\title{
Two component model for hadroproduction in high energy collisions
}

\author{
Alexander Bylinkin* \\ Moscow Institute of Physics and Technology (MIPT), Moscow, Russia \\ E-mail: alexander.bylinkinegmail.com
}

Transverse momentum spectra, $d^{2} \sigma /\left(d \eta d p_{T}^{2}\right)$, of charged hadron production in $p p$-collisions are considered in terms of a recently introduced two component model. The shapes of the particle distributions vary as a function of c.m.s. energy in the collision and the measured pseudorapidity interval. In order to extract predictions on the double-differential cross-sections $d^{2} \sigma /\left(d \eta d p_{T}^{2}\right)$ of hadron production for future LHC-measurements the different sets of available experimental data have been used in this study.

XXVII International Symposium on Lepton Photon Interactions at High Energies

17-22 August 2015

Ljubljana, Slovenia

${ }^{*}$ Speaker. 


\section{Introduction}

Recently a qualitative model considering two sources of hadroproduction has been introduced [1]. It was suggested to parametrize charged particle spectra by a sum of an exponential (Boltzmann-like) and a power-law $p_{T}$ distributions:

$$
\frac{d^{2} \sigma}{d \eta d p_{T}^{2}}=A_{e} \exp \left(-E_{T k i n} / T_{e}\right)+\frac{A}{\left(1+\frac{p_{T}^{2}}{T^{2} \cdot N}\right)^{N}},
$$

where $E_{T k i n}=\sqrt{p_{T}^{2}+M^{2}}-M$ with $M$ equal to the produced hadron mass. $A_{e}, A, T_{e}, T, N$ are free parameters to be determined by a fit to the data. The detailed arguments for this particular choice are given in [1]. The exponential term in this model is associated with thermalized production of hadrons by valence quarks and a quark-gluon cloud coupled to them. The power-law term is related to the mini-jet fragmentation of the virtual partons (pomerons in $\mathrm{pQCD}$ ) exchanged between two colliding partonic systems.

A typical charged particle spectrum as a function of transverse momentum fitted with this function (1.1) is shown in figure 1. As one can see, the exponential term dominates the particle spectrum at low $p_{T}$ values.

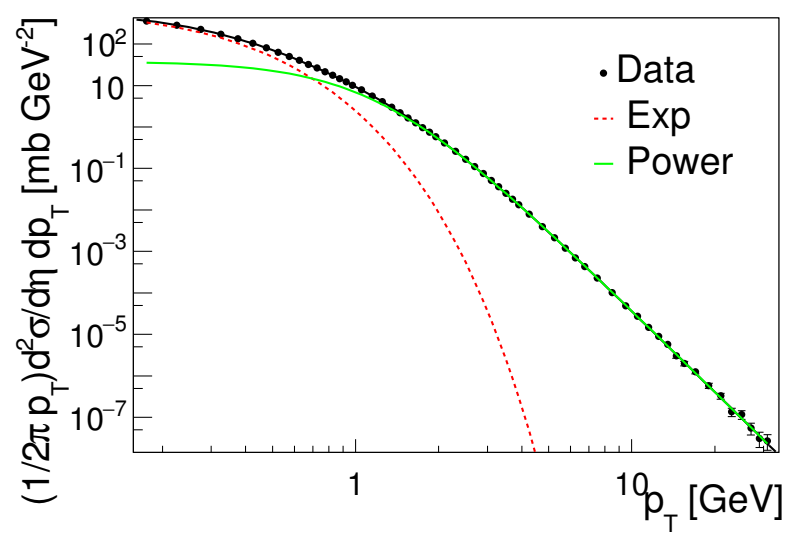

Figure 1: Charge particle differential cross section: the red (dashed) line shows the exponential term and the green (solid) line shows the power law term.

Separating "soft" and "hard" contributions with this model allowed to calculate the predictions on the mean $\left\langle p_{T}\right\rangle$ values as a function of multiplicity in a collision [2] and pseudorapidity distributions of charged particles. However, the major interest of many studies in QCD is the transverse momentum spectrum itself. Therefore, in this article it is discussed how its shape varies in different experiments under various conditions. In [1] it was shown that the parameters of the fit (1.1) show a strong dependence on the collision energy. Unfortunately, due to the fact that different collaborations measure charged particle production in their own phase space and under various experimental configurations, the dependences observed in [1] were smeared and did not allow to make strong predictions for further measurements. Thus, an approach to correct the measurements in order to allow an accurate combination of different experimental data is proposed here. 


\section{Parameter variations}

In [?] it was shown that two sources of hadroproduction described above contribute to different pseudorapidity regions: while the power-law term of (1.1) prevails in the central rapidity region $(|\eta| \lesssim O(1))$, the exponential term dominates at high values of $\eta$. Since each collaboration presents measurements on transverse momentum spectra in various pseudorapidity intervals, these variations might explain the smearing of the dependences in [1]. The idea to study parameter variations as a function of both collision energy and pseudorapidity region has already been successfully tested in [3].

Let us now plot the parameter variations as a function of the rapidity interval between the incoming proton and the produced secondary hadron in the moving proton rest frame according to a simple formula:

$$
\eta^{\prime}=|\eta|-\log \left(\sqrt{s} / 2 m_{p}\right), \quad \eta^{\prime \prime}=|\eta|+\log \left(\sqrt{s} / 2 m_{p}\right)
$$

where $m_{p}$ is the mass of the incoming proton. The results of this procedure are shown in figure 2 . Surprisingly, all the points came to a single line in this interpretation. To understand the origin of this universality one might use Monte $\operatorname{Carlo}(\mathrm{MC})$ generators: hard processes at large $p_{T}$ are known to be described by MC generators pretty well, thus it is expected to get the value of the Nparameter from the fits of the MC-generated spectra rather close to the real data, but with a higher accuracy and in a wider collision energy range. To check this universality, we have produced the Monte Carlo samples for proton-proton collisions at different energies for inelastic(INEL) events with the PYTHIA 8.2 generator. Indeed, the values of the parameter $\mathrm{N}$ extracted from the fits to the MC-generated spectra are nicely placed at the same line. Thus, a universal parameter describing the shape of the transverse momentum spectra in pp-collisions has been found.

Remarkably, similarly to $N$, the $T$ and $T_{e}$ also show dependences as a function of both the collisions energy $\sqrt{s}$ and the measured pseudorapidity interval $\eta$. The variations of the $T$ and $T_{e}$ parameters were studied in [3]. The dependences are shown on figure 2 as well.
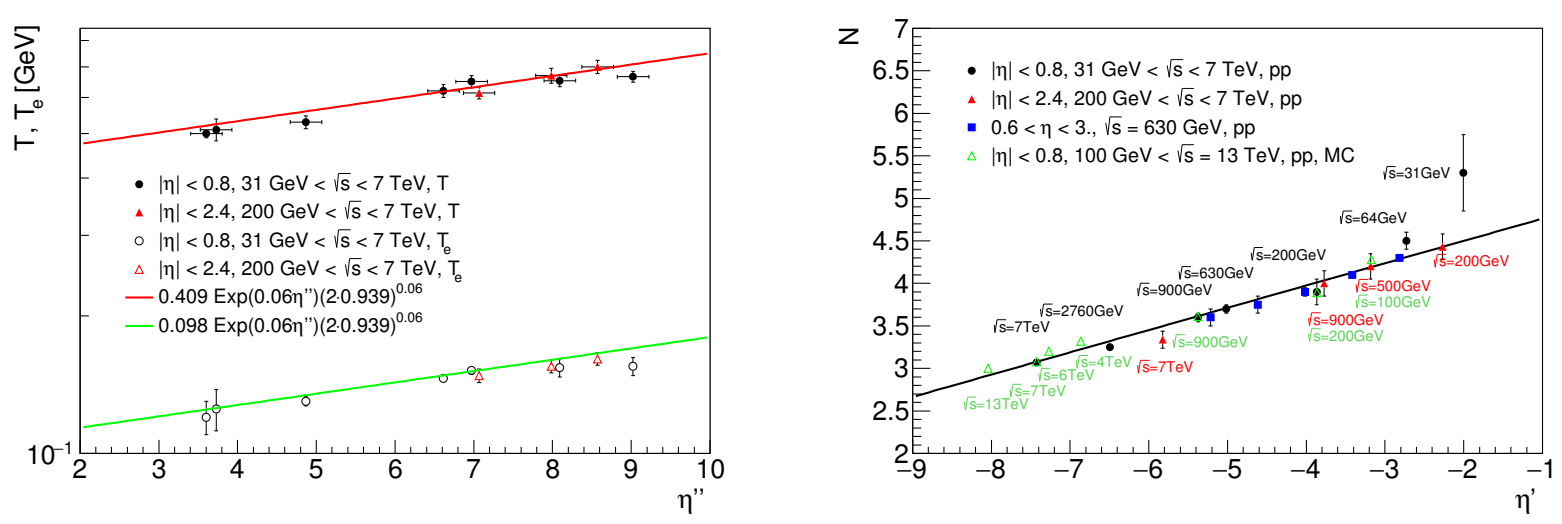

Figure 2: The dependence of the parameters on the pseudorapidity of the secondary hadron in the moving opposite side proton rest frame. 


\section{Prediction for further measurements}

Using the parameter dependences [4] extracted from figure 2, one can calculate double differential cross sections $d^{2} \sigma /\left(d \eta d p_{T}^{2}\right)$ of charged particle production in high energy collisions at different energies for NSD events. These predictions are shown in figure 3 for $|\eta|<0.8$ and $|\eta|<2.4$ pseudorapidity intervals together with the experimental data measured by CMS [5] and ALICE [6]. A good agreement of the prediction with the data can be observed. Thus, these results give us a powerful tool for predicting the spectral shapes in NSD events.

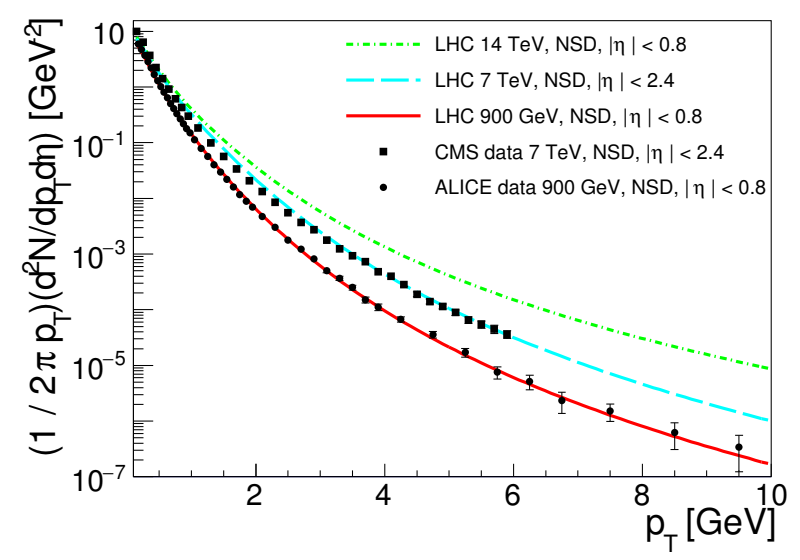

Figure 3: Predictions of the yield of charged particles $\left(1 / 2 \pi p_{T}\right) d^{2} N /\left(d \eta d p_{T}\right)$ in high energy collisions in NSD events together with data points from the ALICE [6] and CMS [5] experiments.

\section{Conclusion}

In conclusion, transverse momentum spectra in $p p$-collisions have been considered using a two component model. Variations of the parameters obtained from the fit have been studied as a function of pseudorapidity $\eta$ and c.m.s. energy $\sqrt{s}$ in the collision. A universal parameter describing a shape of the spectra in pp-collisions was found to be a preudorapidity of a secondary hadron in the moving proton rest frame. Finally, the observed dependences, together with previous investigations allowed to make predictions on double differential spectra $d^{2} \sigma /\left(d p_{T}^{2} d \eta\right)$ at higher energies, successfully tested on the available experimental data.

\section{References}

[1] A. A. Bylinkin and A. A. Rostovtsev, Phys. Atom. Nucl. 75 (2012) 999, arXiv:1008.0332 [hep-ph].

[2] A. A. Bylinkin, M. G. Ryskin, Phys. Rev. D 90 (2014) 1, 017501

[3] A. A. Bylinkin, D. E. Kharzeev and A. A. Rostovtsev, Int. J. Mod. Phys. E 23 (2014) 12, 1450083.

[4] A. Bylinkin, N. S. Chernyavskaya and A. A. Rostovtsev, Eur. Phys. J. C 75 (2015) 4, 166 [arXiv:1501.05235 [hep-ph]].

[5] V. Khachatryan et al. [CMS Collaboration], Phys. Rev. Lett. 105 (2010) 022002 [arXiv:1005.3299 [hep-ex]]. 
[6] K. Aamodt et al. [ALICE Collaboration], Phys. Lett. B 693 (2010) 53-68 [arXiv:1007.0719 [hep-ex]]. 